This is a preprint of: "Dynamics of the FitzHugh-Nagumo system having invariant algebraic surfaces", Jaume Llibre, Yuzhou Tian, Z. Angew. Math. Phys., vol. 72, 15, 2021.

DOI: [10.1007/s00033-020-01450-1]

\title{
DYNAMICS OF THE FITZHUGH-NAGUMO SYSTEM HAVING INVARIANT ALGEBRAIC SURFACES
}

\author{
JAUME LLIBRE ${ }^{1}$ AND YUZHOU TIAN ${ }^{2}$
}

\begin{abstract}
In this paper we study the dynamics of the FitzHugh-Nagumo system $\dot{x}=z, \dot{y}=b(x-d y), \dot{z}=x(x-1)(x-a)+y+c z$ having invariant algebraic surfaces. This system has four different types of invariant algebraic surfaces. The dynamics of the FitzHugh-Nagumo system having two of these classes of invariant algebraic surfaces have been characterized in [21]. Using the quasi-homogeneous directional blow up and the Poincaré compactification, we describe the dynamics of the FitzHugh-Nagumo system having the two remaining classes of invariant algebraic surfaces. Moreover for these FitzHughNagumo systems we prove that they do not have limit cycles.
\end{abstract}

\section{INTRODUCTION}

The FitzHugh-Nagumo system is given by the partical differential system

$$
u_{t}=u_{x x}-f(u)-v, \quad v_{t}=\varepsilon(u-\gamma v),
$$

where $f(u)=u(u-1)(u-a)$, and $0<a<1 / 2, \varepsilon>0, \gamma>0$ are parameters. We say that a bounded solution $(u, v)(x, t)$ of the FitzHugh-Nagumo system (1) with $x, t \in \mathbb{R}$ is a travelling wave if $(u, v)(x, t)=(u, v)(\xi)$, where $\xi=x+c t$ and $c$ is the constant denoting the wave speed. Substituting $u=u(\xi), v=v(\xi)$ into (1), we obtain the ordinary differential system

$$
\begin{aligned}
& \dot{x}=z=P(x, y, z), \\
& \dot{y}=b(x-d y)=Q(x, y, z), \\
& \dot{z}=x(x-1)(x-a)+y+c z=R(x, y, z) .
\end{aligned}
$$

Here the dot denotes derivative with respect to $\xi, x=u, y=v, z=\dot{u}, b=\varepsilon / c$ and $d=\gamma$, see for more details [11].

The FitzHugh-Nagumo system (1) is classical differential system introduced independently by FitzHugh [8] and Nagumo et al. [19]. It is an important model for describing the excitation of neural membranes and the propagation of nerve impulses along an axon. Besides its biological interest, the FitzHugh-Nagumo system has gained wide investigation from the mathematical point of view, such as the existence, uniqueness and stability of its traveling wave solutions, see for instance $[2,9,12-14,19]$, etc.

*Corresponding author: Yuzhou Tian.

2010 Mathematics Subject Classification. Primary 37C10, Secondary 34C05, 37C70.

Key words and phrases. Global dynamics, FitzHugh-Nagumo system, invariant algebraic surface, Poincaré compactification. 
In recent years the FitzHugh-Nagumo system (2) has been investigated from the points of view of its dynamics and integrability. The analytical integrability of the FitzHugh-Nagumo system (2) has been studied by Llibre and Valls in [17]. The Liouvillian integrability of the planar FitzHugh-Nagumo system can be found in [18]. A polynomial $f(x, y, z) \in \mathbb{C}[x, y, z]$ is called a Darboux polynomial for the FitzHugh-Nagumo system (2) if

$$
P \frac{\partial f}{\partial x}+Q \frac{\partial f}{\partial y}+R \frac{\partial f}{\partial z}=k f
$$

for some polynomial $k(x, y, z) \in \mathbb{C}[x, y, z]$, which is called the cofactor of $f$. If $f$ is a Darboux polynomial of system (2), then $f=0$ is an invariant algebraic surface of system (2), because if an orbit of system (2) has a point on the surface $f=0$ the whole orbit is contained in such a surface. This follows easily from the definition of Darboux polynomial.

The following result completes the classification of all the invariant algebraic surfaces of the FitzHugh-Nagumo system, see [22].

Theorem 1. There are four types of generators for the invariant algebraic surfaces of the FitzHugh-Nagumo system (2), and two polynomial first integrals when $b=$ $c=0$, see Table 1 .

Table 1: The generators of FitzHugh-Nagumo system (2)

\begin{tabular}{|c|c|c|}
\hline Darboux polynomials & Cof & Parameters \\
\hline$f_{1}=\frac{1}{2} x^{4}-z^{2}+2 x y+\frac{2}{3} c x z+\left(\frac{1}{9} c^{2}-1\right) x^{2}$ & $\frac{4}{3} c$ & $\begin{array}{l}a=-1, b d=-c, c \neq 0 \\
b=\frac{2}{27} c^{3}-\frac{1}{3} c\end{array}$ \\
\hline$f_{2}=\frac{1}{2} x^{4}-z^{2}+2 x y+\frac{2}{3} c x z+\left(\frac{1}{9} c^{2}-1\right) x^{2}-\frac{1}{2} d y^{2}$ & $\frac{4}{3} c$ & $\begin{array}{l}a=-1, b d=-\frac{2}{3} c, c \neq 0 \\
b=\frac{2}{27} c^{3}-\frac{1}{3} c\end{array}$ \\
\hline $\begin{array}{l}f_{3}=\frac{1}{2} x^{4}-z^{2}+2 x y+\frac{2}{3} c x z-\frac{2}{3}(a+1) x^{3} \\
+\left(\frac{1}{9} c^{2}+a\right) x^{2}-\frac{2}{9} c(a+1) z-\frac{2}{3}(a+1) y \\
-\frac{2}{27} c^{2}(a+1) x\end{array}$ & $\frac{4}{3} c$ & $\begin{array}{l}a \neq-1, b d=-c, c \neq 0, \\
b=\frac{2}{27} c^{3}-\frac{1}{9} a^{2} c+\frac{1}{9} a c-\frac{1}{9} c, \\
2 c^{2}+3 a^{2}-12 a+3=0\end{array}$ \\
\hline $\begin{array}{l}f_{4}=\frac{1}{2} x^{4}-z^{2}-\frac{1}{2} d y^{2}+2 x y+\frac{2}{3} c x z-\frac{2}{3}(a+1) x^{3} \\
+\left(\frac{1}{9} c^{2}+a\right) x^{2}-\frac{2}{9} c(a+1) z-\frac{1}{3}(a+1) y \\
-\frac{2}{27} c^{2}(a+1) x\end{array}$ & $\frac{4}{3} c$ & $\begin{array}{l}a \neq-1, b d=-\frac{2 c}{3}, c \neq 0, \\
b=\frac{2}{27} c^{3}-\frac{1}{9} a^{2} c+\frac{1}{9} a c-\frac{1}{9} c, \\
2 c^{2}+a^{2}-7 a+1=0\end{array}$ \\
\hline$f_{5}=y$ & 0 & $b=c=0$ \\
\hline$f_{6}=\frac{1}{4} x^{4}-\frac{1}{2} z^{2}-\frac{1}{3}(a+1) x^{3}+x y+\frac{1}{2} a x^{2}$ & 0 & $b=c=0$ \\
\hline
\end{tabular}

A nonconstant function $H(x, y, z, t): \mathbb{R}^{3} \times \mathbb{R} \rightarrow \mathbb{C}$ is called an invariant of the FitzHugh-Nagumo system (2) if $H(x, y, z, t)$ is a constant along each trajectory $(x(t), y(t), z(t))$ of system (2). If $H$ is independent of the time $t$, it is a first integral. If $H$ is of the form $f(x, y, z) \exp (-\sigma t)$ with $f \in \mathbb{R}[x, y, z]$ and $\sigma$ a non-zero constant, then $H$ is called a Darboux invariant. Note that $H=f(x, y, z) \exp (-\sigma t)$ is a Darboux invariant if and only if $f$ is a Darboux polynomial with cofactor $\sigma$. From Theorem 1 we summarize the results on the integrability and on the existence of Darboux invariants for system (2) in the next theorem, for a proof see [22]. 
Theorem 2. The FitzHugh-Nagumo system (2) has the Darboux invariants I and the first integrals $H$ given in Table 2.

Table 2: Darboux invariants and first integrals of system (2)

\begin{tabular}{|c|c|}
\hline Darboux polynomials & Parameters \\
\hline (a) $\begin{array}{l}a=-1, b d=-c, c \neq 0 \\
b=\frac{2}{27} c^{3}-\frac{1}{3} c\end{array}$ & $I=\left(\frac{1}{2} x^{4}-z^{2}+2 x y+\frac{2}{3} c x z+\left(\frac{1}{9} c^{2}-1\right) x^{2}\right) \exp \left(-\frac{4}{3} c t\right)$ \\
\hline (b) $\begin{array}{l}a=-1, b d=-\frac{2}{3} c, c \neq 0 \\
b=\frac{2}{27} c^{3}-\frac{1}{3} c\end{array}$ & $I=\left(\frac{1}{2} x^{4}-z^{2}+2 x y+\frac{2}{3} c x z+\left(\frac{1}{9} c^{2}-1\right) x^{2}-\frac{1}{2} d y^{2}\right) \exp \left(-\frac{4}{3} c t\right)$ \\
\hline $\begin{array}{l}a \neq-1, b d=-c, c \neq 0, \\
b=\frac{2}{27} c^{3}-\frac{1}{9} a^{2} c+\frac{1}{9} a c-\frac{1}{9} c, \\
2 c^{2}+3 a^{2}-12 a+3=0\end{array}$ & $\begin{array}{l}I=\left(\frac{1}{2} x^{4}-z^{2}+2 x y+\frac{2}{3} c x z-\frac{2}{3}(a+1) x^{3}\right. \\
+\left(\frac{1}{9} c^{2}+a\right) x^{2}-\frac{2}{9} c(a+1) z-\frac{2}{3}(a+1) y \\
\left.-\frac{2}{27} c^{2}(a+1) x\right) \exp \left(-\frac{4}{3} c t\right)\end{array}$ \\
\hline $\begin{array}{l}a \neq-1, b d=-\frac{2 c}{3}, c \neq 0, \\
b=\frac{2}{27} c^{3}-\frac{1}{9} a^{2} c+\frac{1}{9} a c-\frac{1}{9} c, \\
2 c^{2}+a^{2}-7 a+1=0\end{array}$ & $\begin{array}{l}I=\left(\frac{1}{2} x^{4}-z^{2}-\frac{1}{2} d y^{2}+2 x y+\frac{2}{3} c x z-\frac{2}{3}(a+1) x^{3}\right. \\
+\left(\frac{1}{9} c^{2}+a\right) x^{2}-\frac{2}{9} c(a+1) z-\frac{1}{3}(a+1) y \\
\left.-\frac{2}{27} c^{2}(a+1) x\right) \exp \left(-\frac{4}{3} c t\right)\end{array}$ \\
\hline (e) $b=c=0$ & $\begin{array}{l}H_{1}=y \\
H_{2}=\frac{1}{4} x^{4}-\frac{1}{2} z^{2}-\frac{1}{3}(a+1) x^{3}+x y+\frac{1}{2} a x^{2}\end{array}$ \\
\hline
\end{tabular}

In this paper we classify the dynamics of FitzHugh-Nagumo system (2) having an invariant algebraic surface. For parameter $c \neq 0$, we can assume $c>0$, because $c<$ 0 can be reduced to $c>0$ doing the change of variables $(x, y, z, t) \mapsto(x, y,-z,-t)$.

Since the FitzHugh-Nagumo system (2) is a polynomial differential system in $\mathbb{R}^{3}$ we can compactify it in order to study its dynamics in a neighborhood of the infinity doing the Poincaré compactification. Roughly speaking the Poincaré compactification consists in identify $\mathbb{R}^{3}$ with the interior of the closed ball $\mathbb{B}^{3}$ centered at the origin of $\mathbb{R}^{3}$ and radius one, called the Poincaré ball. Then the boundary of the ball $\mathbb{B}^{3}$, i.e. the sphere $\mathbb{S}^{2}$, is identified with the infinity of $\mathbb{R}^{3}$, and called the Poincaré sphere. In $\mathbb{R}^{3}$ we can go to infinity in as many directions as points has the sphere $\mathbb{S}^{2}$. Then the FitzHugh-Nagumo system (2) can be extended analytically to the Poincaré ball $\mathbb{B}^{3}$, For more details on the Poincaré compactification and the expression of the extended differential systems in the different local charts of $\mathbb{B}^{3}$, see for instance [5].

The following theorem is the first main result of this paper.

Theorem 3. For all values of the parameters $a, b, c, d \in \mathbb{R}$ the phase portrait of the FitzHugh-Nagumo system (2) on the Poincaré sphere is topologically equivalent to the one of Figure 1. Then system (2) has the circle defined by the boundary of the plane $x=0$ at infinity filled up of singular points, and there are no additional singular points at the sphere of infinity in the Poincaré compactification.

The dynamics of system (2) with the invariant algebraic surfaces $f_{1}=0$ and $f_{2}=0$ have been characterized in [21]. We only need to characterize the dynamics of system (2) with the invariant algebraic surfaces $f_{3}=0$ and $f_{4}=0$. 


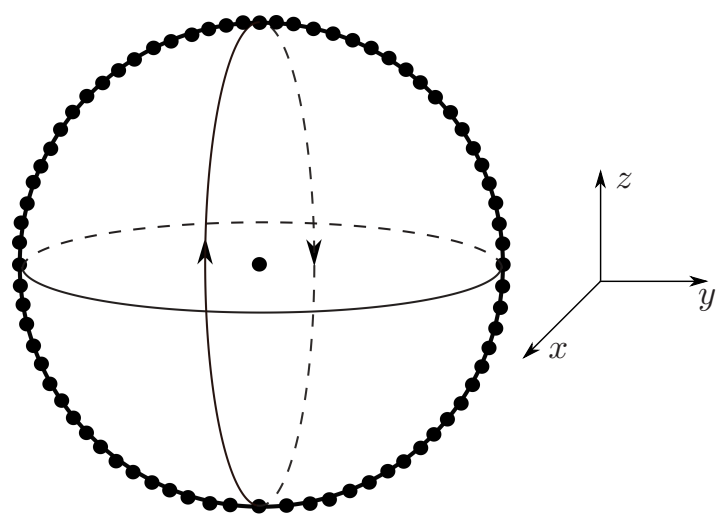

Figure 1. Phase portrait at infinity on the Poincaré ball of system (2).

Theorem 4. For $c>0$, the FitzHugh-Nagumo system (2) with the invariant algebraic surfaces $f_{3}=0$ and $f_{4}=0$ have the following dynamics.

(a) The boundary at infinity of the surface $f_{3}=0$ at infinity is the great circle $\{x=0\} \cap\left\{x^{2}+y^{2}+z^{2}=1\right\}$ filled up with singular points. The dynamics of system (2) on the invariant algebraic surface $f_{3}=0$ are characterized by Figure 4 .

(b) The boundary at infinity of the surface $f_{4}=0$ at infinity is the great circle $\{x=0\} \cap\left\{x^{2}+y^{2}+z^{2}=1\right\}$ filled up with singular points. The dynamics of system (2) on the invariant algebraic surface $f_{4}=0$ are characterized by either Figure 7 or 9 .

The paper is organized as follows. We present some preliminary results in section 2. The proofs of Theorems 3 and 4 will be given in sections 3 and 4 , respectively.

\section{Preliminary Results}

In this section we recall some results that we shall need for proving our theorems.

Lemma 5. Let $f(x, y, z)=0$ be an invariant algebraic surface of the differential system (2) with the constant cofactor $k \neq 0$, and let $I=f(x, y, z) e^{-k t}$ be its associated Darboux invariant. Assume that $\phi(t)=(x(t), y(t), z(t))$ is a solution of system (2) not contained in the invariant surface $f(x, y, z)=0$.

(a) If $k>0$ the $\omega$-limit of $\phi(t)$ inside the Poincaré ball $\mathbb{B}^{3}$ is contained in the sphere of Poincaré $\mathbb{S}^{2}$ intersection with the closure of the surface $f(x, y, z)=0$, and the $\alpha$-limit of $\phi(t)$ inside the Poincaré ball $\mathbb{B}^{3}$ is contained in the closure of the surface $f(x, y, z)=0$.

(b) If $k<0$ then statement (a) holds interchanging the $\omega$-limit by the $\alpha$-limit.

The proof of Lemma 5 is given in Proposition 5 of [16]. In fact there it is proved for polynomial differential systems in $\mathbb{R}^{2}$, but that proof works for polynomial differential systems in $\mathbb{R}^{n}$. To describe the dynamics of the FitzHugh-Nagumo system (2) having an invariant algebraic surface, by Lemma 5 , we only need to investigate it on the invariant surface. 
Lemma 6. Let $f(x, y, z)=0$ be an algebraic invariant surface of degree $m$ in $\mathbb{R}^{3}$. The extension of this surface to the boundary of the Poincaré ball is given by the equations

$$
w^{m} f\left(\frac{x}{w}, \frac{y}{w}, \frac{z}{w}\right)=0, \quad w=0 .
$$

A proof of Lemma 6 can be found in Lemma 2.1 of [15].

For studying the local phase portrait of the singular points of a two-dimensional differential systems whose linear part is identically zero we shall use the quasihomogeneous directional blow up (or $(\alpha, \beta)$-blow up) technique, see Chapter 3 of [7] or $[1,6]$ for more details. The quasi-homogeneous directional blow up coordinate change can be written as

positive $x$-direction: $(x, y) \mapsto\left(\bar{u}^{\alpha}, \bar{u}^{\beta} \bar{v}\right), \quad$ negative $x$-direction: $(x, y) \mapsto\left(-\bar{u}^{\alpha}, \bar{u}^{\beta} \bar{v}\right)$, positive $y$-direction: $(x, y) \mapsto\left(\bar{u} \bar{v}^{\alpha}, \bar{v}^{\beta}\right)$, negative $y$-direction: $(x, y) \mapsto\left(\bar{u} \bar{v}^{\alpha},-\bar{v}^{\beta}\right)$, where $(\alpha, \beta) \in \mathbb{N}^{+} \times \mathbb{N}^{+}$. Using the Newton diagram we can determine the values of $\alpha$ and $\beta$, see [3] and [4] for more details.

A phase portrait of a differential system defined in an open set $U$ of $\mathbb{R}^{n}$ is the decomposition of $U$ as the union of all the orbits of the differential system.

We say that two phase portraits one defined on the open set $U$ and the other defined in the open set $V$ are topologically equivalent if there exists a homeomorphism $h: U \rightarrow V$ which send the orbits on $U$ onto the orbits of $V$, preserving or reversing the orientation of all the orbits.

The separatrices of a differential system or vector field on a surface $S$ are the singular points, the limit cycles and the separatrices of all its hyperbolic sectors. The set $\Sigma$ all separatrices of a vector field on $S$ is a closed set. The open connected components of $S \backslash \Sigma$ are called canonical regions. A separatrix configuration $\Sigma^{*}$ of a vector field on $S$ is the union of $\Sigma$ with one orbit in each canonical region.

Let $\Sigma_{1}^{*}$ and $\Sigma_{2}^{*}$ two separatrices configurations of two vector fiels on the surface $S$. We say that $\Sigma_{1}^{*}$ and $\Sigma_{2}^{*}$ are topologically equivalent if there exists a homeomorphism $h: \Sigma_{1}^{*} \rightarrow \Sigma_{2}^{*}$ which send the orbits on $\Sigma_{1}^{*}$ onto the orbits of $\Sigma_{2}^{*}$, preserving or reversing the orientation of all the orbits.

Neumann provided the following theorem in [20].

Theorem 7 (Neumann's Theorem). Two continuous flows on a surface $S$ with isolated singular points are topologically equivalent if and only if their separatrix configurations are topologically equivalent.

This theorem shows that the phase portrait of a differential system on a surface is determined by its separatrix configurations if all the singular points of the differential system are isolated.

The following result provides necessary and sufficient conditions in order that all the roots of a polynomial $g(z) \in \mathbb{R}[z]$ have negative real parts, see page 231 of [10].

Theorem 8 (Routh-Hurwitz Criterion). All roots of the real polynomial $g(z)=$ $a_{0} z^{n}+a_{1} z^{n-1}+\cdots+a_{n-1} z+a_{n}\left(a_{0}>0\right)$ have negative real parts if and only if

$$
\Delta_{1}>0, \Delta_{2}>0, \ldots, \Delta_{n}>0,
$$


where

$$
\Delta_{i}=\operatorname{det}\left(\begin{array}{cccccc}
a_{1} & a_{3} & a_{5} & \cdots & & \\
a_{0} & a_{2} & a_{4} & \cdots & & \\
0 & a_{1} & a_{3} & \cdots & & \\
0 & a_{0} & a_{2} & a_{4} & & \\
\vdots & \vdots & \vdots & \vdots & \ddots & \\
& & & & & a_{i}
\end{array}\right)\left(a_{k}=0 \text { if } k>n\right)
$$

is the Hurwitz determinant of order $i(i=1,2, \cdots, n)$.

Corollary 9. All roots of the real polynomial $a_{0} z^{3}+a_{1} z^{2}+a_{2} z+a_{3}\left(a_{0}>0\right)$ have negative real parts if and only if

$$
\Delta_{1}=a_{1}>0, \Delta_{2}=a_{1} a_{2}-a_{3} a_{0}>0, a_{3}>0 .
$$

Lemma 10. If system (2) has the invariant algebraic surface $f_{4}=0$, then

$$
E_{1}=\left(\frac{a+1}{3}, \frac{(a-2)(a+1)(2 a-1)}{27}, 0\right)
$$

is singular point, which is a local repeller.

Proof. Note that the parameters $a, b, c, d$ satisfy $c>0, d=9 /((a-2)(2 a-1))$, $b=-2 c /(3 d)=-2(a-2)(2 a-1) c / 27, c^{2}=-\left(a^{2}-7 a+1\right) / 2$ and $(7-3 \sqrt{5}) / 2<$ $a<(7+3 \sqrt{5}) / 2$ (see Table 1). With these conditions system (2) becomes

$$
\begin{aligned}
& \dot{x}=z, \\
& \dot{y}=-\frac{2}{27}(a-2)(2 a-1) c x+\frac{2}{3} c y, \\
& \dot{z}=x(x-1)(x-a)+y+c z .
\end{aligned}
$$

It is easy to check that $E_{1}$ is a singular point of system (3). After the time reversal system (3) can be rewritten as

$$
\begin{aligned}
& \dot{x}=-z, \\
& \dot{y}=\frac{2}{27}(a-2)(2 a-1) c x-\frac{2}{3} c y, \\
& \dot{z}=-x(x-1)(x-a)-y-c z .
\end{aligned}
$$

The Jacobian matrix of system (4) at $E_{1}$ is

$$
J=\left(\begin{array}{ccc}
0 & 0 & -1 \\
\frac{2}{27}(a-2)(2 a-1) c & -\frac{2}{3} c & 0 \\
\frac{1}{3}\left(a^{2}-a+1\right) & -1 & -c
\end{array}\right)
$$

The characteristic equation of matrix $J$ is $27 \lambda^{3}+45 c \lambda^{2}+9\left(a^{2}-a+2 c^{2}+1\right) \lambda+$ $2(a+1)^{2} c=0$. Using the notations of Lemma 10 we have $45 c>0,2(a+1)^{2} c>0$ and $\Delta_{2}=27 c\left(13 a^{2}-19 a+30 c^{2}+13\right)=-54\left(a^{2}-43 a+1\right) c$. Since $c>0$ and $(7-3 \sqrt{5}) / 2<a<(7+3 \sqrt{5}) / 2$, then $\Delta_{2}>0$. By Corollary 9 all th eigenvalues of $E_{1}$ have negative real parts. So the singular point $E_{1}$ of system (4) is a local attractor. This means that $E_{1}$ is a local repeller for system (2). This ends the proof. 


\section{Proof of Theorem 3}

Poincaré introduced his compactification for polynomial vector fields in $\mathbb{R}^{2}$. For the extension of the Poincaré compactification to polynomial vector fields in $\mathbb{R}^{n}$ see [5]. Using the results of [5] for the Poincaré compactification in $\mathbb{R}^{3}$ we get that in the local chart $U_{1}$ the Poincaré compactified system (2) becomes

$$
\begin{aligned}
& \dot{u}=w^{2}(b-b d u-v u), \\
& \dot{v}=1-(a+1) w+a w^{2}+c v w^{2}+u w^{2}-v^{2} w^{2}, \\
& \dot{w}=-v w^{3} .
\end{aligned}
$$

On the invariant plane $w=0$ (the infinity in $U_{1}$ ) system (6) reduces to

$$
\dot{u}=0, \quad \dot{v}=1
$$

This system has no singular points.

In the local chart $U_{2}$ the Poincaré compactified system (2) writes

$$
\begin{aligned}
& \dot{u}=w^{2}\left(v+b d u-b u^{2}\right), \\
& \dot{v}=u^{3}-(a+1) u^{2} w+w^{2}+(a-b v) u w^{2}+(b d+c) v w^{2}, \\
& \dot{w}=b w^{3}(d-u) .
\end{aligned}
$$

On the invariant plane $w=0$ (again the infinity in $U_{2}$ ) system (8) becomes

$$
\dot{u}=0, \quad \dot{v}=u^{3} .
$$

The singular points of system (9) filled up the straight line $u=0$.

Once we have studied the singular points on the local charts $U_{1}$ and $U_{2}$ the unique additional singular point at infinity can be the origin of the local chart $U_{3}$. Of course, at infinity all the diametrically points with respect to the origin of $\mathbb{B}^{3}$ of the singular points of the charts $U_{k}$ for $k=1,2,3$ are also infinite singular points.

In the local chart $U_{3}$ system (2) is given by

$$
\begin{aligned}
& \dot{u}=-u^{4}+(a+1) u^{3} w+w^{2}-a u^{2} w^{2}-(c+v) u w^{2}, \\
& \dot{v}=-u^{3} v+(a+1) u^{2} v w-v^{2} w^{2}+(b-a v) u w^{2}-(b d+c) v w^{2}, \\
& \dot{w}=-u^{3} w+(1+a) u^{2} w^{2}-c w^{3}-a u w^{3}-v w^{3} .
\end{aligned}
$$

So the origin of $U_{3}$ is also a singular point.

In summary, taking into account the definition of the local charts $U_{k}$ for $k=$ $1,2,3$ and their symmetric charts $V_{k}$ for $k=1,2,3$ (see [5], or in particular [15]) all the infinite singular points of the FitzHugh-Nagumo system (2) filled up the boundary of the plane $x=0$ at infinity in the Poincaré sphere.

This and the flows on the local charts $U_{1}$ and $V_{1}$ completes the proof of Theorem 3

\section{Proof of Theorem 4}


4.1. System (1) with the invariant algebraic surface $f_{3}=0$. In this case system (2) has the invariant algebraic surface

$f_{3}=\frac{x^{4}}{2}-\frac{2}{3}(a+1) x^{3}+\left(a+\frac{c^{2}}{9}\right) x^{2}+\frac{2 c x z}{3}+2 x y-z^{2}-\frac{2}{27}(a+1) c^{2} x-\frac{2}{9}(a+1) c z-\frac{2}{3}(a+1) y=0$.

Since $2 c^{2}+3 a^{2}-12 a+3=0$, then $2-\sqrt{3}<a<2+\sqrt{3}$. By Lemma 6 the boundary of this surface in the Poincaré sphere $\mathbb{S}^{2}$, i.e. at the infinity of $\mathbb{R}^{3}$, is described by the equations

$\frac{x^{4}}{2}-\frac{2}{27}(a+1)\left(c^{2} x+3 c z+9 y\right) w^{3}+\frac{1}{9}\left(9 a x^{2}+c^{2} x^{2}+6 c x z+18 x y-9 z^{2}\right) w^{2}-\frac{2}{3}(a+1) x^{3} w=0$, and $w=0$, that is, by $x=0$ and $w=0$. This means that the boundary of this surface at infinity is the circle filled up with infinite singular points.

Define

$$
\begin{aligned}
& V_{1}=\left\{(x, y, z): f_{3}(x, y, z)=0,3 x-a-1=0\right\}, \\
& V_{2}=\left\{(x, y, z): f_{3}(x, y, z)=0,3 x-a-1 \neq 0\right\} .
\end{aligned}
$$

The set $V_{1}$ reduces to $162 z^{2}+(a+1)^{2}\left(2 c^{2}+3 a^{2}-12 a+3\right)=0$, i.e. to the straight line intersection of the planes $3 x-a-1=0$ and $z=0$, because $2 c^{2}+3 a^{2}-12 a+3=0$. Therefore system (2) restricted to the straight line $V_{1}$ becomes

$$
\dot{y}=b\left(\frac{a+1}{3}-d y\right) \text {. }
$$

In the surface $V_{2}$ we have

$y(x, z)=\frac{36(a+1) x^{3}-27 x^{4}-6\left(9 a+c^{2}\right) x^{2}+4 c x(a c+c-9 z)+6 z(2(a+1) c+9 z)}{36(3 x-a-1)}$,

and system (2) restricted to this surface is

$$
\begin{aligned}
& \dot{x}=z, \\
& \dot{z}=\frac{x(3 x-2 a-2)\left(27 x^{2}-18 x-18 a x+18 a-2 c^{2}\right)+24 c(3 x-a-1) z+54 z^{2}}{36(3 x-a-1)} .
\end{aligned}
$$

Under the condition $2 c^{2}+3 a^{2}-12 a+3=0$ the above system reduces to

$$
\begin{aligned}
& \dot{x}=z, \\
& \dot{z}=\frac{1}{12}\left(x(3 x-a-1)(3 x-2-2 a)+8 c z+\frac{18 z^{2}}{3 x-a-1}\right) .
\end{aligned}
$$

After a rescaling of the time variable $d \xi=12(3 x-a-1) d \tau$, system (11) becomes

$$
\begin{aligned}
& x^{\prime}=12 z(3 x-a-1), \\
& y^{\prime}=x(3 x-a-1)^{2}(3 x-2 a-2)+8 c(3 x-a-1) z+18 z^{2},
\end{aligned}
$$

where now the prime denotes derivative with respect to $\tau$.

Using the Poincaré compactification in $\mathbb{R}^{2}$ (see for instance Chapter 5 of [7]) we investigate the infinite singular points of system (12). Doing the change to the local chart $U_{1}$ given by $x=1 / v, y=u / v$ and rescaling the time $d s=v^{4} d \tau$ system (12) can be written as

$$
\begin{aligned}
& u^{\prime}=27-2(a+1)\left((a+1)^{2}+4 c u-6 u^{2}\right) v^{3}+3\left(5(a+1)^{2}+8 c u-6 u^{2}\right) v^{2}-36(a+1) v, \\
& v^{\prime}=12 u v^{3}(a v+v-3) .
\end{aligned}
$$


On the $u$-axis system (13) has no singular points.

Now we write system (12) in the local chart $U_{2}$ using the change $x=u / v, y=$ $1 / v, d s=v^{4} d \tau$ to get

$$
\begin{aligned}
& \dot{u}=2(a+1)\left((a+1)^{2} u^{2}+4 c u-6\right) v^{3}+3\left(6-5(a+1)^{2} u^{2}-8 c u\right) u v^{2}+36(a+1) u^{4} v-27 u^{5}, \\
& \dot{v}=2(a+1)\left(4 c+(a+1)^{2} u\right) v^{4}-3\left(5(a+1)^{2} u^{2}+8 c u+6\right) v^{3}+36(a+1) u^{3} v^{2}-27 u^{4} v .
\end{aligned}
$$

The linear part of the origin of system (14) is identically zero. We use the quasihomogeneous blow up $(u, v) \mapsto\left(\bar{u}, \bar{u}^{2} \bar{v}\right)$ in the positive $u$-direction. After division by $\bar{u}^{4}$, we obtain

$\dot{\bar{u}}=-3 \bar{u}\left(8 c \bar{u} \bar{v}^{2}-6 \bar{v}^{2}+9\right)$,

$\dot{\bar{v}}=27 \bar{v}-54 \bar{v}^{3}+12(2 c \bar{v}+3 a+3) \bar{u} \bar{v}^{2}+(a+1)(8 c \bar{v}-15(a+1)) \bar{u}^{2} \bar{v}^{3}+2(a+1)^{3} \bar{u}^{3} \bar{v}^{4}$.

On $\{\bar{u}=0\}$ system $(15)$ has a saddle at $(0,0)$ and two stable nodes at $(0, \pm 1 / \sqrt{2})$.

Consider the blow-up $(u, v) \mapsto\left(-\bar{u}, \bar{u}^{2} \bar{v}\right)$ in the negative $u$-direction. After cancelling a common factor $\bar{u}^{4}$, we have

(16)

$$
\begin{aligned}
& \dot{\bar{u}}=3 \bar{u}\left(8 c \bar{u} \bar{v}^{2}+6 \bar{v}^{2}-9\right), \\
& \dot{\bar{v}}=27 \bar{v}-54 \bar{v}^{3}-12(2 c \bar{v}+3 a+3) \bar{u} \bar{v}^{2}+(a+1)(8 c \bar{v}-15(a+1)) \bar{u}^{2} \bar{v}^{3}-2(a+1)^{3} \bar{u}^{3} \bar{v}^{4} .
\end{aligned}
$$

On the line $\bar{u}=0$, system (16) has a saddle at $(0,0)$ and two stable nodes at $(0, \pm 1 / \sqrt{2})$.

We perform blow-up $(u, v) \mapsto\left(\bar{u} \bar{v}, \bar{v}^{2}\right)$ in the positive $v$-direction as well as $(u, v) \mapsto\left(\bar{u} \bar{v},-\bar{v}^{2}\right)$ in the negative $v$-direction. After division by $\bar{v}^{4}$, we obtain respectively

$$
\begin{aligned}
& \dot{\bar{u}}=27 \bar{u}-\left((a+1)^{3} \bar{v}^{2}+12 c\right) \bar{u}^{2} \bar{v}-4(a+1) c \bar{v}^{2} \bar{u}-18(a+1) \bar{u}^{4} \bar{v}+\frac{15}{2}(a+1)^{2} \bar{u}^{3} \bar{v}^{2}-\frac{27}{2} \bar{u}^{5}, \\
& \dot{\bar{v}}=-9 \bar{v}+(a+1)\left(4 c-\frac{15}{2}(a+1) \bar{u}^{2}\right) \bar{v}^{3}+6\left(3(a+1) \bar{u}^{2}-2 c\right) \bar{u} \bar{v}^{2}+(a+1)^{3} \bar{u} \bar{v}^{4}-\frac{27}{2} \bar{u}^{4} \bar{v},
\end{aligned}
$$

and

$$
\begin{aligned}
& \dot{\bar{u}}=27 \bar{u}+\left((a+1)^{3} \bar{v}^{2}-12 c\right) \bar{v} \bar{u}^{2}+4(a+1) c \bar{v}^{2} \bar{u}+18(a+1) \bar{u}^{4} \bar{v}+\frac{15}{2}(a+1)^{2} \bar{u}^{3} \bar{v}^{2}-\frac{27}{2} \bar{u}^{5}, \\
& \dot{\bar{v}}=-9 \bar{v}-(a+1)\left(\frac{15}{2}(a+1) \bar{u}^{2}+4 c\right) \bar{v}^{3}-6\left(3(a+1) \bar{u}^{2}+2 c\right) \bar{u} \bar{v}^{2}-(a+1)^{3} \bar{u} \bar{v}^{4}-\frac{27}{2} \bar{u}^{4} \bar{v} .
\end{aligned}
$$

The origins of systems (17) and (18) are saddles. The blow up quasi-homogeneous procedure and the local phase portrait of system (14) at the origin are described in Figure 2 .

System (12) has three singular points: the origin, $e_{1}=(2(a+1) / 3,0)$ and $e_{2}=((a+1) / 3,0)$. The origin is a saddle because its eigenvalues are $-2(a+$ 1) $\left(2 c \pm \sqrt{4 c^{2}+6(a+1)^{2}}\right)$. The eigenvalues of $e_{1}$ are $2(a+1)\left(2 c \pm \sqrt{4 c^{2}+6(a+1)^{2}}\right)$, so $e_{1}$ is a saddle. The linear part of $e_{2}$ is identically zero. Moving the singular point 


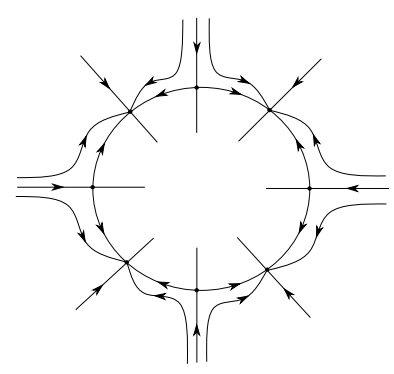

(1) Blow up.

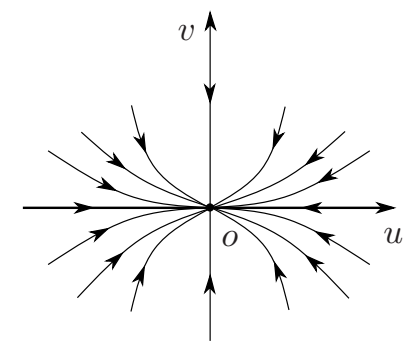

(2) Local phase portrait.

Figure 2. The local phase portrait of system (14) at the origin.

$e_{2}$ to the origin through the change $(x, z) \mapsto(x+(a+1) / 3, z)$, system (12) can be written as

$$
\dot{x}=36 x z, \quad \dot{z}=3\left(9 x^{4}-(a+1)^{2} x^{2}+8 c x y+6 y^{2}\right) .
$$

Applying (1,1)-type quasi-homogeneous directional blow up, we get that the origin of system (19) has the local phase portrait given in Figure 3.

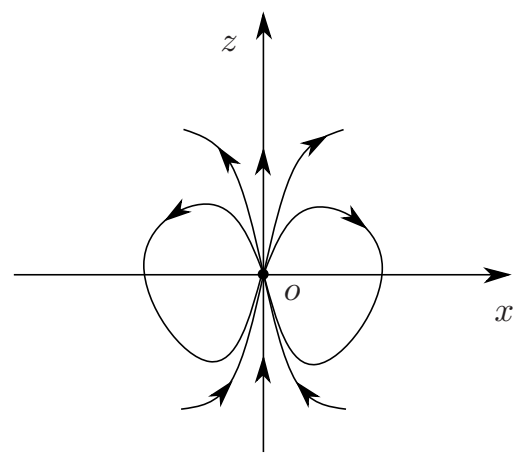

Figure 3. Local phase portrait of system (19) at the origin.

In summary on the invariant surface $f_{3}=0$ we have the phase portrait in the Poincaré disc of system (11) in Figure 4.

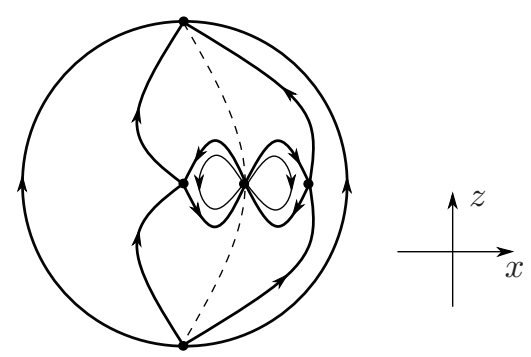

FiguRE 4. Phase portrait for system (11). The dashed straight line is $3 x-a-1=0$, where the system is not defined. 
4.2. System (1) with the invariant algebraic surface $f_{4}=0$. System (1) has the invariant algebraic surface

$$
\begin{aligned}
f_{4}= & \frac{x^{4}}{2}-\frac{2}{3}(a+1) x^{3}+\left(a+\frac{c^{2}}{9}\right) x^{2}+\frac{2 c x z}{3}-\frac{d y^{2}}{2}+2 x y-z^{2}-\frac{2}{27}(a+1) c^{2} x \\
& -\frac{2}{9}(a+1) c z-\frac{1}{3}(a+1) y=0 .
\end{aligned}
$$

Using Lemma 6 the boundary of this surface at the Poincaré sphere of the infinity is given by the intersection of the surfaces

$$
\begin{aligned}
& \frac{x^{4}}{2}-\frac{2}{3}(a+1) x^{3} w+w^{2}\left(a x^{2}+\frac{c^{2} x^{2}}{9}+\frac{2 c x z}{3}-\frac{d y^{2}}{2}+2 x y-z^{2}\right) \\
& -\frac{1}{27}(a+1) w^{3}\left(2 c^{2} x+6 c z+9 y\right)=0,
\end{aligned}
$$

and $w=0$, that is $x=0$ and $w=0$. So the boundary of this surface at infinity is again the circle of the infinity filled up with singular points.

From the invariant algebraic surface $f_{4}=0$ we get

$$
z^{ \pm}(x, y)=\frac{1}{18}(2 c(3 x-a-1) \pm \sqrt{2 \eta})
$$

where

(20)

$$
\begin{aligned}
\eta(x, y)= & 2 c^{2}\left(18 x^{2}-12(a+1) x+(a+1)^{2}\right)+27\left(\left(3 x^{2}-4(a+1) x+6 a\right) x^{2}\right. \\
& \left.-2(a-6 x+1) y-3 d y^{2}\right) .
\end{aligned}
$$

System (2) restricted to the surface $f_{4}=0$ becomes

$$
\dot{x}=z^{ \pm}(x, y), \quad \dot{y}=b(x-d y) .
$$

Let $\left(x_{0}, y_{0}\right)$ be a singular point of system (21). Then $x_{0}=d y_{0}$ and $z^{ \pm}\left(x_{0}, y_{0}\right)=0$. We note that $f_{4}\left(x_{0}, y_{0}, z^{ \pm}\left(x_{0}, y_{0}\right)\right)=f_{4}\left(d y_{0}, y_{0}, 0\right)=0$, that is,

$$
\frac{1}{54}\left(27 d^{4} y_{0}^{3}-36(a+1) d^{3} y_{0}^{2}+3 d\left(2 d\left(9 a+c^{2}\right)+27\right) y_{0}-2\left(2 c^{2} d+9\right)(a+1)\right) y_{0}=0 \text {. }
$$

The singular points of system (21) are characterized by equation (22). The parameters $a, b, c, d$ satisfy $c>0, d=9 /\left(2 a^{2}-5 a+2\right), b=-2 c /(3 d)=-2(a-2)(2 a-$ 1) $c / 27, c^{2}=-\left(a^{2}-7 a+1\right) / 2$ and $(7-3 \sqrt{5}) / 2<a<(7+3 \sqrt{5}) / 2$ (see Table 1). Equation $(22)$ can be reduced to

$$
y_{0}\left(27 y_{0}-4 a^{3}+6 a^{2}+6 a-4\right)\left(27 y_{0}-2 a^{3}+3 a^{2}+3 a-2\right)^{2}=0 .
$$

The solutions of this equation are

$$
y_{0}=0, y_{1}=\frac{1}{27}(a-2)(a+1)(2 a-1) \text { and } y_{2}=\frac{2}{27}(a-2)(a+1)(2 a-1) \text {. }
$$

Let $e_{0}=(0,0), e_{1}=\left(d y_{1}, y_{1}\right)$ and $e_{2}=\left(d y_{2}, y_{2}\right)$, that is,

$e_{1}=\left(\frac{a+1}{3}, \frac{(a-2)(a+1)(2 a-1)}{27}\right)$ and $e_{2}=\left(\frac{2(a+1)}{3}, \frac{2(a-2)(a+1)(2 a-1)}{27}\right)$.

Doing the adequate computations, we have

$$
z^{+}\left(e_{0}\right)=z^{ \pm}\left(e_{1}\right)=z^{-}\left(e_{2}\right)=0 .
$$


Consider the subsystems of system (21)

$$
\dot{x}=z^{+}(x, y)=\frac{1}{18}(2 c(3 x-a-1)+\sqrt{2 \eta}), \quad \dot{y}=b(x-d y) .
$$

From equation (23), system (24) has two finite singular points $e_{0}$ and $e_{1}$. The Jacobian matrix of system (24) is

$$
J(x, y)=\left(\begin{array}{cc}
\frac{1}{18}\left(6 c+\frac{\partial_{x} \eta}{\sqrt{2 \eta}}\right) & \frac{\partial_{y} \eta}{18 \sqrt{2 \eta}} \\
b & -b d
\end{array}\right) .
$$

Since $\operatorname{det}\left(J\left(e_{0}\right)\right)=-(a+1)^{2} / 9<0, e_{0}$ is a saddle. The Jacobian matrix $J(x, y)$ at the singular point $e_{1}$ is not defined at $e_{1}$ because of $\eta\left(e_{1}\right)=0$. This means that system $(24)$ is not analytic at $e_{1}$. In order to get local phase portrait of system (24) at $e_{1}$, we go back to investigate system (2). From Lemma 10 it follows that the restriction of the singular point $E_{1}$ to the surface $f_{4}=0$ becomes the singular point $e_{1}$ of system (24), which is repeller.

Assume that system (24) has a limit cycle. Then the limit cycle surrounding the singular point $e_{1}$ of system (24) must intersect the line $x=(a+1) / 3$, see Figure 5 . The flow of system $(24)$ over the line $x=(a+1) / 3$ are given by

$$
\left.\dot{x}\right|_{x=\frac{a+1}{3}}=\left.\frac{\sqrt{2 \eta}}{18}\right|_{x=\frac{a+1}{3}}=\frac{1}{9} \sqrt{-\frac{\left(27 y-2 a^{3}+3 a^{2}+3 a-2\right)^{2}}{2(a-2)(2 a-1)}} \geq 0,
$$

which is in contradiction with the limit cycle turning clockwise in positive time. Thus, system (24) has no limit cycles.

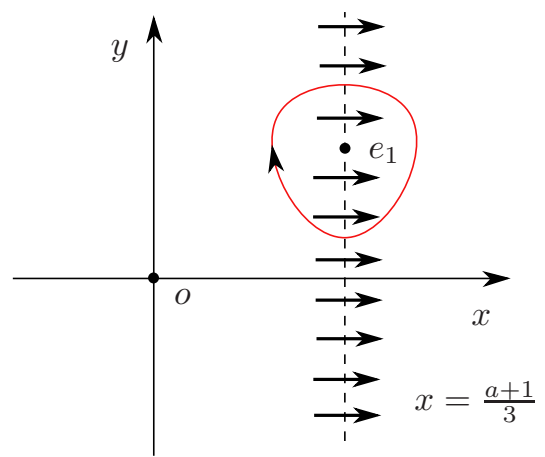

FiguRE 5. Flow of system (24) over the straight line $x=(a+1) / 3$.

Taking the Poincaré transformation $x=1 / v, y=u / v$ and rescaling the time $d t=v^{2} d \tau$, system (24) becomes

$$
\begin{aligned}
& \dot{u}=b v+u\left(\frac{c}{9}(a+1) v^{2}+\frac{c}{3} v-\frac{\sqrt{2 \delta}}{18}\right), \\
& \dot{v}=-\frac{\sqrt{2 \delta}}{18} v-\frac{c}{3} v^{2}+\frac{c}{9}(a+1) v^{3},
\end{aligned}
$$

where

$\delta=2(a+1)^{2} c^{2} v^{4}-6(a+1)\left(4 c^{2}+9 u\right) v^{3}+9\left(18 a+4 c^{2}-9 u(d u-4)\right) v^{2}-108(a+1) v+81$. 
On the $u$-axis the origin of system (26) is the unique singular point which is a stable node.

Doing the Poincaré transformation $x=u / v, y=1 / v$ with the scaling $d t=v^{2} d \tau$, we obtain

$$
\begin{aligned}
& \dot{u}=\frac{\sqrt{2 \delta_{1}}}{18}-\frac{c}{9}(a+1) v^{2}-\frac{c}{3} u v-b u^{2} v, \\
& \dot{v}=-\left(\frac{2}{3} c+b u\right) v^{2},
\end{aligned}
$$

where

$$
\begin{aligned}
\delta_{1}= & 81 u^{4}-108(a+1) u^{3} v+9\left(2\left(9 a u+2 c^{2} u+18\right) u-9 d\right) v^{2}-6(a+1)\left(4 c^{2} u+9\right) v^{3} \\
& +2(a+1)^{2} c^{2} v^{4} .
\end{aligned}
$$

The origin of system (27) is degenerate. With the help of (1,2)-type quasi-homogeneous directional blow up, we obtain that the local phase portrait of system (27) at origin is topologically equivalent to Figure 6 .

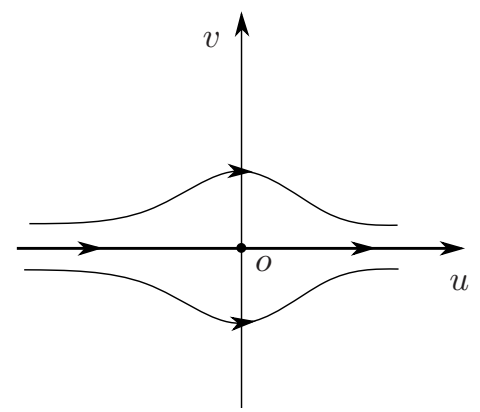

FiguRE 6. Local phase portrait of system (27) at the origin.

Based on the above discussions the phase portrait of system (24) in the Poincaré disc is described in Figure 7.

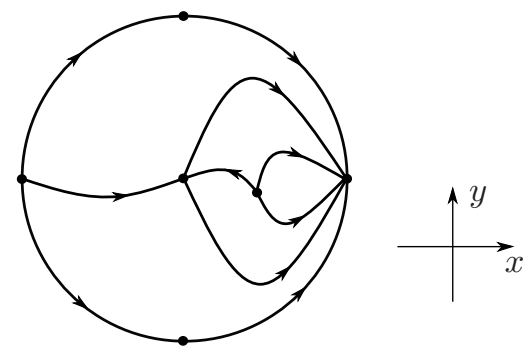

Figure 7. Phase portrait for system (24).

For subsystems of system (21)

$$
\dot{x}=z^{-}(x, y)=\frac{1}{18}(2 c(3 x-a-1)-\sqrt{2 \eta}), \quad \dot{y}=b(x-d y),
$$


it has two singular points $e_{2}$ and $e_{1}$. The Jacobian matrix of system (24) is

$$
J(x, y)=\left(\begin{array}{cc}
\frac{1}{18}\left(6 c-\frac{\partial_{x} \eta}{\sqrt{2 \eta}}\right) & -\frac{\partial_{y} \eta}{18 \sqrt{2 \eta}} \\
b & -b d
\end{array}\right) .
$$

Since $\operatorname{det}\left(J\left(e_{2}\right)\right)=-(a+1)^{2} / 9<0, e_{2}$ is a saddle. System (29) is also not analytic at $e_{1}$. By Lemma 10, the singular point $e_{1}$ is repeller. In the same way as system, system (29) has no limit cycles.

Using the Poincaré transformation $x=1 / v, y=u / v$ and rescaling the time $d t=v^{2} d \tau$, system (28) becomes

$$
\begin{aligned}
& \dot{u}=b v+u\left(\frac{c}{9}(a+1) v^{2}+\frac{c}{3} v+\frac{\sqrt{2 \delta}}{18}\right), \\
& \dot{v}=\frac{\sqrt{2 \delta}}{18} v-\frac{c}{3} v^{2}+\frac{c}{9}(a+1) v^{3},
\end{aligned}
$$

with

$\delta=2(a+1)^{2} c^{2} v^{4}-6(a+1)\left(4 c^{2}+9 u\right) v^{3}+9\left(18 a+4 c^{2}-9 u(d u-4)\right) v^{2}-108(a+1) v+81$.

On the $u$-axis, the origin is the unique singular point of system (30), which is an unstable node.

Making the Poincaré transformation $x=u / v, y=1 / v$ with the scaling $d t=$ $v^{2} d \tau$, system (28) can be written as

$$
\begin{aligned}
& \dot{u}=-\frac{\sqrt{2 \delta_{1}}}{18}-\frac{c}{9}(a+1) v^{2}-\frac{c}{3} u v-b u^{2} v, \\
& \dot{v}=-\left(\frac{2}{3} c+b u\right) v^{2},
\end{aligned}
$$

with

$$
\begin{aligned}
\delta_{1}= & 81 u^{4}-108(a+1) u^{3} v+9\left(2\left(9 a u+2 c^{2} u+18\right) u-9 d\right) v^{2}-6(a+1)\left(4 c^{2} u+9\right) v^{3} \\
& +2(a+1)^{2} c^{2} v^{4} .
\end{aligned}
$$

The origin of system (31) is degenerate. Doing $(1,2)$-type quasi-homogeneous directional blow up, the local phase portrait of system (31) at origin is topologically equivalent to Figure 8.

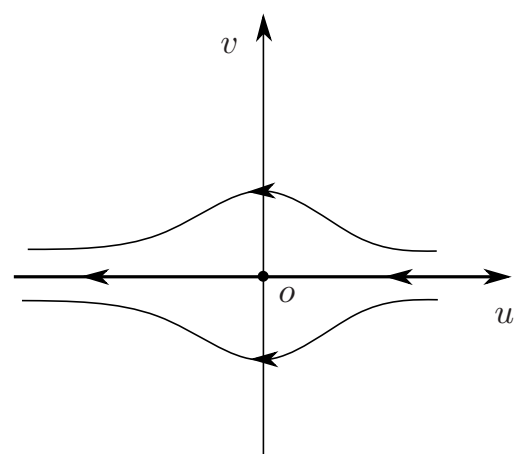

FiguRE 8. Local phase portrait of system (31) at the origin. 
So the phase portrait of system (28) is given in Figure 9. From Theorem 4 we know that the phase portraits of systems (24) and (28) are topologically equivalent. In fact the phase portraits of systems (24) and (28) describe the dynamics of system (2) on the surface $f_{4}=0$ projected onto the $(x, y)$-plane.

This completes the proof of Theorem 4.

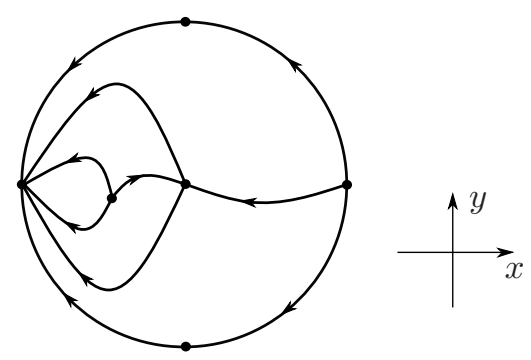

Figure 9. Phase portrait for system (28).

\section{ACKNOWLEDGEMENTS}

The first author is partially supported by the Ministerio de Ciencia, Innovación y Universidades, Agencia Estatal de Investigación grants M TM2016-77278-P (FEDER), the Agència de Gestió d'Ajuts Universitaris i de Recerca grant 2017SGR1617, and the H2020 European Research Council grant MSCA-RISE-2017-777911. The second author is partially supported by the National Natural Science Foundation of China (No. 11971495 and No. 11801582), China Scholarship Council (No. 201906380022) and Guangdong Basic and Applied Basic Research Foundation (No. 2019A1515011239).

\section{REFERENCES}

[1] M. Álvarez, A. Ferragut, and X. Jarque, A survey on the blow up technique, Internat. J. Bifur. Chaos Appl. Sci. Engrg., 21 (2011), 3103-3118.

[2] G. Arioli And H. KoCH, Existence and stability of traveling pulse solutions of the FitzHughNagumo equation, Nonlinear Anal., 113 (2015), 51-70.

[3] A. Briuno, Local methods in nonlinear differential equations, Springer, 1989.

[4] M. Brunella, M. Miari, Topological equivalence of a plane vector field with its principal part defined through Newton polyhedra, J. Differential Equations, 85 (1990), 338-366.

[5] A. Cima, J. Llibre, Bounded polynomial vector fields, Trans. Amer. Math. Soc., 318 (1990), 557-579

[6] F. Dumortier, Techniques in the theory of local bifurcations: Blow-up, normal forms, nilpotent bifurcations, singular perturbations, in Bifurcations and periodic orbits of vector fields, Springer, 1993, 19-73.

[7] F. Dumortier, J. Llibre, J. Artés, Qualitative theory of planar differential systems, Springer, 2006.

[8] R. FitzHugh, Impulses and physiological states in theoretical models of nerve membrane, Biophysical journal, 1 (1961), 445.

[9] G. Flores, Stability analysis for the slow travelling pulse of the FitzHugh-Nagumo system, SIAM J. Math. Anal., 22 (1991), 392-399.

[10] F. R. Gantmacher, Applications of the theory of matrices, Interscience Publishers Ltd., London, 1959.

[11] W. GaO, J. WAng, Existence of wavefronts and impulses to FitzHugh-Nagumo equations, Nonlinear Anal., 57 (2004), 667-676. 
[12] H. J. Hupkes, B. SAndstede, Stability of pulse solutions for the discrete FitzHugh-Nagumo system, Trans. Amer. Math. Soc., 365 (2013), 251-301.

[13] C. K. R. T. Jones, Stability of the travelling wave solution of the FitzHugh-Nagumo system, Trans. Amer. Math. Soc., 286 (1984), 431-469.

[14] W. Liu, E. VAN VLECK, Turning points and traveling waves in FitzHugh-Nagumo type equations, J. Differential Equations, 225 (2006), 381-410.

15] J. Llibre, M. Messias, P. R. DA Silva, Global dynamics of the Lorenz system with invariant algebraic surfaces, Internat. J. Bifur. Chaos Appl. Sci. Engrg., 20 (2010), 3137-3155.

[16] J. Llibre, R. D. S. OliveIRA, Quadratic systems with invariant straight lines of total multiplicity two having Darboux invariants, Commun. Contemp. Math., 17 (2015), 1450018, 17.

[17] J. Llibre, C. Valls, Analytic first integrals of the FitzHugh-Nagumo systems, Z. Angew. Math. Phys., 60 (2009), 237-245.

[18] J. Llibre, C. VAlls, Liouvillian integrability of the FitzHugh-Nagumo systems, J. Geom. Phys., 60 (2010), 1974-1983.

[19] J. Nagumo, S. Arimoto, and S. Yoshizawa, An active pulse transmission line simulating nerve axon, Proceedings of the IRE, 50 (1962), 2061-2070.

[20] D. Neumann, Classification of continuous flows on 2-manifolds, Proc. Amer. Math. Soc., 48 (1975), 73-81

[21] C. VALLS, On the global dynamics of the Newell-Whitehead system, J. Nonlinear Math. Phys., 26 (2019), 569-578.

[22] L. Zhang, J. Yu, Invariant algebraic surfaces of the FitzHugh-Nagumo system, J. Math. Anal. Appl., 483 (2020), 123097, 19.

1 Departament de Matemátiques, Universitat Autónoma de Barcelona, 08193 Bellaterra, Barcelona, Catalonia, Spain

E-mail address: jllibre@mat.uab.cat

2 School of Mathematics (Zhuhai), Sun Yat-Sen University, Zhuhai 519082, P.R. China

E-mail address: tianyzh3@mail2.sysu.edu.cn 